\title{
The Origin of Non-chaotic Behavior in Identically Driven Systems.
}

\author{
P. M. Gade and Chaitali Basu \\ International Centre for Theoretical Physics \\ P.O. Box 586, Trieste 34100, ITALY
}

\begin{abstract}
Recently it has been found that different physical systems driven by identical random noise behave exactly identical after a long time. It is also suggested that this is an outcome of finite precision in numerical experiments. Here we show that the origin of the non-chaotic behavior lies in the structural instability of the attractor of these systems which changes to a stable fixed point for strong enough drive. We see this to be true in all the systems studied in literature. Thus we affirm that in chaotic systems, synchronization can not occur only by addition of noise unless the noise destroys the strange attractor and the system is no longer chaotic.
\end{abstract}

Of late there has been considerable attention drawn to the problem in which the behavior of an ensemble of initial conditions obeying the same laws of motion and driven by an identical sequence of random forces start following the same random trajectory asymptotically.

This is observed in several distinct cases. Fahy and Hamann [1] observed that if a particle obeying Newton's equations (without friction) in a potential $V$ is stopped at regular time intervals $\tau$ and all its velocity components are reset to random values, then for a given sequence of random values asymptotic trajectories become identical for all initial conditions. This a stronger statement than the observation that the statistical distribution of trajectories become independent of initial conditions. This phenonomenon occurs for the choice of a time interval $\tau$ lower than a threshold value $\tau_{c}$. They remark that this is common for all bounded systems.

Another observation of a similar phenomenon is due to Maritan and Banavar [2]. In this letter, they show that a pair of chaotic systems driven by identical noise of sufficient strength have identical trajectories asymptotically. 
They studied the noisy logistic map and the Lorenz system. They have also given an analytic argument for the above phenomenon in terms of Langevin dynamics [2]. It was pointed out by Pikovsky [3] that the phenomenon of synchronization in logistic maps does not occur in numerical experiments in quadrupole precision. Thus he has suggested that the synchronization in systems with positive Lyapunov exponent is a numerical effect of the insufficient precision of calculations. Maritan and Banavar [4 replied that a pair of chaotic system subjected to the same noise has higher probability of becoming identical compared to the case in absence of noise.

The observation by Pikovsky raises some fundamental questions. The question is whether all the above reported observations are indeed physical phenomena or just an artifact of finite precision of calculations in which two trajectories that come close within the precision of numerical calculations stick together. This question is important since there has been an attempt to understand systems like globally coupled maps using maps driven by identical random noise [5]. These systems have more experimental relevance [6]. In this system of globally coupled maps, it is observed that though the maps form clusters at a particular precision, the clustering disappears at higher precision [6]. This clustering is also observed in globally coupled Josephson Junction arrays (JJA) [7]. It is difficult to know whether this clustering in JJA will change with changing precision as the original calculations themselves are in double precision and it is difficult to demand higher accuracy. In short, it questions the reliability of numerical computations in this and similar phenomena which are of higher physical relevance.

One more question that arises in this context is how vital is the presence of randomness for such synchronisation?

In this letter, we show that this synchronization phenomenon is indeed physical in certain cases. It is an effect of the fact that the strange attractor of the undriven system is replaced by a stable fixed point for strong enough perturbation. When the attractor is a stable fixed point, different trajectories converge exponentially towards it. If the parameter is now driven randomly with the system flipping between chaotic and non-chaotic regimes, and if the overall rate of contraction of the trajectories is higher than that of the divergence in the chaotic regime, the trajectories will converge for all precisions. Here we point out that the randomness is of marginal significance and even if the drive is periodic or constant in nature the phenomenon of synchronisation occurs. 
We stress that the statement of 'synchronization of chaotic systems subject to same random noise' is not correct as the trajectories no longer remain chaotic. We illustrate this statement by the example of the Lorenz system.

The numerical experiment in [1] can be formulated in one-dimension as

$$
\begin{gathered}
x((n+1) \tau)=G(x(n \tau), v(n \tau)) \\
v(n \tau)=r_{n}
\end{gathered}
$$

where $r_{n}$ s are delta correlated random numbers chosen from some distribution and $G(x, v)$ is a function which gives the final position of the particle starting from $(x, v)$ and undergoing motion in the potential $V(x)$ for time $\tau$.

To check the essentiality of randomness in this example we set all $r_{n} \mathrm{~s}$ to a constant $\mu$, for the one dimensional Duffing potential $V(x)=x^{4}-x^{2}$ as studied in [1]. We observe that for smaller values of $\mu$, there is a high probability that the system relaxes to a stable fixed point for $\tau=0.5$. In other words the point attractor at the fixed point of $G$, is relatively stable for smaller values of $\mu$, though there is no clear critical point below which the fixed point is always stable. We define a range $I_{s}=\left[-\mu_{c}(\tau), \mu_{c}(\tau)\right]$, such that the trajectories do not converge to a fixed point outside this range. In Fig. 1, we give a plot of the coarse grained probability that a fixed point is stable as a function of $\mu$ for various values of $\tau$. As can be seen from the plot, it is more and more unlikely to have a stable fixed point for higher values of $\mu$. This trend is more pronounced for higher values of $\tau$. Now it is clear that if one chooses the $r_{n}$ s from the range of values for which there is high probability that the fixed point is stable, the trajectories will converge. The fact that randomness is of marginal significance is checked by putting $r_{n}=4 \cos (\delta n), \delta=0.001$ for $\tau=.5$ where we still observe the convergence of trajectories $\left(\mu_{c}(.5) \simeq 3.7\right)$. This is due to the fact that the fixed point changes only continuously with $\mu$ and the trajectories keep on getting attracted exponentially. The range $I_{s}$ will also determine the average rate of convergence. However, even if the the overall rate diverges, but the the random number $r_{n}$ takes succesive values in the regime $I_{s}$ for a long enough time, the trajectories may come close together within numerical precision and virtually converge. It may not be possible to see this convergence in numerical experiments with higher precision and thus in these cases one may observe a precision dependent convergence. 
Here we point out the importance of the requirement of boundedness in these systems. In bounded systems the existance of the fixed point for $G(x, v)$ in Eq. 11 with $r_{n}$ s set to constant $\mu$ is guaranteed [12]. Now the question is why it is always stable for small values of $\tau$ ? It can be argued the following way. The trajectories move towards the minimum of the potential energy with some of the potential energy getting converted into kinetic energy. The successive reassignment of the velocity to a reasonably small value after each time interval $\tau$ forces the system to dissipate some part of the kinetic energy and hence also the total energy. This trend continues till the particle comes near the potential minimum. Here the motion of the particle gets bounded within a part of the phase space near the potential minimum. The fixed point $x_{0}(\mu)$ must be in this bounded region. The stability of this point is given by $\frac{d G\left(x_{0}(\mu), \mu\right)}{d x}=1-\frac{d^{2} V}{d x^{2}} \frac{\tau^{2}}{2}$ in the small time limit. This quantity is less than unity if the second derivative of the potential is positive which is certain if the fixed point is near the potential minimum. (Potential minimum is a stable fixed point for the case of zero velocity for any choice of $\tau$ and since the position of the fixed point $x_{0}(\mu)$ as well as $\frac{d G\left(x_{0}(\mu), \mu\right)}{d x}$ vary smoothly with $\mu$, we expect the stability to be retained for small enough velocities.) Thus we explain the stability of the fixed point for small enough times and velocities. However for large times and velocities the behavior that is true at the potential minimum may not continue. One can easily see this for an exactly solvable case of harmonic oscillator where $G$ is known and it can be checked that there is no critical time $\tau_{c}$ except that for the choice of $\tau$ s such that $\omega \tau=n \pi$, the fixed point becomes marginally stable and convergence of trajectories is not possible. In this case of harmonic oscillator, $\frac{d G\left(x_{0}, \mu_{0}\right)}{d x} \leq 1$ always. This explains the factors determining the critical time $\tau_{c}$ above which the trajectories no longer converge.

Now we consider the example considered in [2]. The Lorenz system [8] is considered which is defined as

$$
\begin{aligned}
& \frac{d x}{d t}=P(y-x)=f(x, y, z) \\
& \frac{d y}{d t}=-x z+m x-y=g(x, y, z) \\
& \frac{d z}{d t}=x y-b z=h(x, y, z) .
\end{aligned}
$$

with $\mathrm{P}=10, \mathrm{~b}=8 / 3$ and $\mathrm{m}=28$, which is integrated with time step of 0.001 unit. The $y$ equation is evolved as

$$
y(t+\Delta t)=y(t)+\int_{t}^{t+\Delta t} g(x, y, z) d t+r_{t} W \sqrt{\Delta t} .
$$


where $r_{t}$ is an uniform random number in range $[0,1]$. For higher accuracy we have chosen to use Runge-Kutta method with adoptive stepsize control instead of Euler scheme used in ref. [2]. For this system, Ref. [2] claims that above a critical value of $W=W_{c}=2 / 3$ the synchronisation occurs.

As in the case of [1], here also we check the necessity of randomness for the synchronisation by replacing the random number $r_{t}$ by unity and try to check the behavior for different $W \mathrm{~s}$. We see that for $W>W_{+} \simeq 0.16$ or for $W<W_{-} \simeq-0.16$, the fixed point is stable. This can be checked by following it using Newton-Raphson method and calculating eigenvalues and also by perturbing a little about the fixed point and checking whether the system relaxes back to it. Thus one has a stable chaotic behavior only in the range $I_{c}=\left[W_{-}, W_{+}\right]$. Now in case when the system is driven out of this regime $I_{c}$ by random forces, the effective lyapunov exponent of the system becomes negative. As in earlier case, it will depend on the time that the trajectories spend in the region $I_{c}$ and outside it. In this case, the trajectories can clump together if the lyapunov exponent is negative. We point out that the negative lyapunov exponent is necessary for this clumping.

The transient time required for clumping grows as we decrease the value of $W$. However, we observe that within our computation time and precision the critical value of $W$ is much less than the one quoted in the text as $W_{c}=2 / 3$ which could be the result of more precise integration scheme and longer computation times. Although, this quantitative correction is of marginal significance, we would like to point out that the statement in ref. [2] that, 'However, the strange attractor is not replaced by anything simple such as a fixed point or limit cycle.' is misleading since the strange attractor is indeed replaced by a simpler structure at least in the parameter range outside $I_{c}$. We also point out that the largest lyapunov exponent from the linearised equations of the variables of the system, computed along the noisy trajectory, is negative in the parameter regime of convergence. Thus the trajectories are no longer chaotic in the regime of convergence. To check that it is indeed so, we do computations for large enough times as the system is nonstationary and short-time computation can give a spurious indication that the lyapunov exponent has converged to a positive value.

In Fig. 2 we plot the largest lyapunov exponent as a function of constant $W$ calculated at time $t=6.410^{4}$. We observe a sudden drop around $W=.19$ at which the lyapunov exponent becomes negative. This value could be slightly lower for larger computation times. This explains the clumping in 
case when the random number in Eq. 3 has higher mean, i.e. the value of $W$ is higher.

It is mentioned in [2] that the phenomenon is not observed if the random numbers $r_{t} \mathrm{~s}$ are symmetric around 0 . This assertion is quite correct since the $y$ component of stable fixed point changes its sign with the sign of $W$ and there is a discontinuity in its stability in $I_{c}$ regime. We note here that the stability of the fixed point dictates the behavior of trajectories only in its close vicinity. In this local neighborhood the trajectories get attracted to the fixed point. However, this picture will not be true for the points that are not in its neighborhood. Thus this local convergence will not be valid for the points which are away from the fixed point. The fixed point is changing discontinuously and considerably in this case. Thus due to the fact that the perturbation is being continuously applied, trajectories may never come close enough to either fixed points simultaneously. This is the reason why one will not observe the collapse of trajectories whereas it is possible in the case when the stable fixed point changes only continuously.

We measure the lyapunov exponent for the synchronised case and see that it is indeed negative in the regime in which synchronisation is observed. Thus the trajectories are no longer chaotic.

Here we also point out that similar situation can not arise in systems in which such structural instability is not expected. However, the case of precision dependent synchronization in logistic maps has a different origin. In [2], they have given an example of noisy logistic maps to illustrate their point of view. However, when we repeated the similar experiment for the case of noisy tent maps, we did not observe this effect. This is an artifact of the fact that tent map does not have locally negative lyapunov exponent at any point 9 .

This synchronisation phenomenon is also observed in Ref [10 in which they showed that in a random dynamical system variation of a parameter causes a transition from a situation where an initial cloud of particles eventually permanently clumps at a point to a situation where the particles are eventually distributed on a fractal.

We would point out that the results in [10] do not clash with our observations. Here the collapse of trajectories occur when the lyapunov exponent is negative and this is expected. They study the following two dimensional model

$$
x_{n+1}=\left[x_{n}+\left(1-e^{-\alpha}\right) \alpha^{-1} y_{n}\right] \bmod (2 \pi)
$$




$$
y_{n+1}=e^{-\alpha} y_{n}+k \sin \left(x_{n+1}+\theta_{n}\right)
$$

where $\theta_{n}$ s are independent random variables with uniform probability density in $0 \leq \theta \leq 2 \pi$. In this model, for the parameter range in question, the fixed point is always stable in the absence of noise. If one replaces noise by a constant still the same behavior is observed except that the position of the fixed point changes. However, for lower values of the parameter $\alpha$ dictating the dissipation rate, the continuous random forcing kicks the system out of the basin of attraction of the fixed point and the trajectories never clump together. Thus though the trajectories go to the fixed point always in the absence of noise, for noise of strength comparable to the dissipation, the trajectories do not clump. Here dissipation forces the system towards the fixed point and randomness drives it away from it. These act as competing parameters. Thus it is possible that the trajectories that would otherwise converge do not converge owing to random noise. We point out that in the limit of strong dissipation the parameter range in which the clumping occurs is the same as the parameter range in which the fixed point is stable [11].

However, in this letter we discuss the reverse phenomenon, namely of chaotic trajectories converging by random perturbations. We show that it is possible only if these perturbations change the basic nature of the system and one has a stable nonchaotic attractor for higher perturbations.

In conclusion, we have tried to trace the origin of the phenomenon of clumping observed in different systems which are driven by identical random forces. We have shown that it is due to the structural instability in the system which results from large perturbations. We have also tried to explain the conditions under which the precision dependent clumping could occur. Here we note that the trajectories converge and clump since these perturbations force it to a state in which system has a negative lyapunov exponent. Thus the system is no longer chaotic. The important factors are dissipation and forcing whereas randomness does not play a significant role. We have also discussed the reverse phenomenon, namely the one in which the trajectories that would otherwise go to the stable fixed point display positive lyapunov exponent. Thus we do not see any clumping provided the noise is high and dissipation is comparatively low.

We acknowledge discussions with Prof. Pikovsky, Prof. Maritan and Prof. Cerdeira and a helpful correspondence with Prof. Banavar. We also thank Prof. Pikovsky for sharing [3] prior to publication. 


\section{References}

[1] S. Fahy and D. R. Hamman, Phys. Rev. Lett., 76169 (1992).

[2] A. Maritan and J. R. Banavar, Phys. Rev. Lett., 1451, 72 (1994).

[3] A. S. Pikovsky, Phys. Rev. Lett., 2931, 73 (1994).

[4] A. Maritan and J. R. Banavar, Phys. Rev. Lett., 2932, 73 (1994).

[5] S. Sinha, Phys. Rev. Lett.,69, 3306 (1992).

[6] K. Kaneko, Physica 41D, 137 (1990).

[7] D. Dominguez and H.A. Cerdeira, Phys. Rev. Lett. 71, 3359 (1993).

[8] E. N. Lorenz, J. Atmos. Sci. 20, 130 (1963).

[9] P. M. Gade and A. S. Pikovsky (In preparation).

[10] L. Yu, E. Ott and Q. Chen, Phys. Rev. Lett., 293565 (1990)

[11] L. Yu, E. Ott and Q. Chen, Physica 53D (1991)

[12] Since at $\infty, x((n+1) \tau)<x(n \tau)$ and at $-\infty x(n \tau)<x((n+1) \tau)$, the continuous function $G$ must have $x(n \tau)=x((n+1) \tau)$ for at least one point. 


\section{Figure Captions}

Fig. 1 The coarse grained probability that the trajectories converge to a fixed point as function of velocity $\mu$ (i.e. setting $r_{n}=\mu$ for Eq. 11) for different values of $\tau$. The curves from above to below represent $\tau=$ $.005, .05, .1, .2, .3, .4$ and .5 respectively.

Fig. 2 The largest lyapunov exponent $\lambda$ as a function of $W$. 